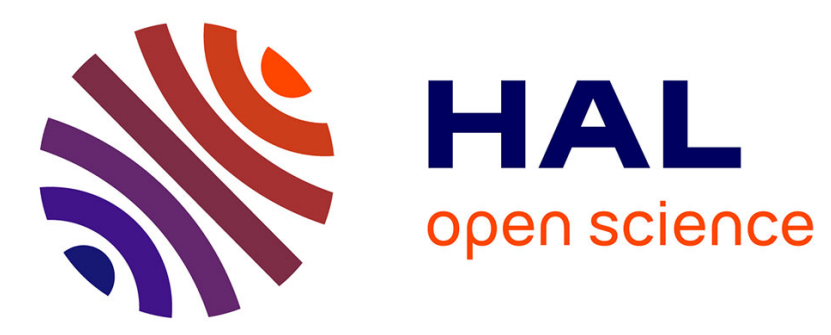

\title{
Identifier et caractériser un genre: l'exemple des interviews politiques
}

Mylène Blasco-Dulbecco, Paul Cappeau

\section{To cite this version:}

Mylène Blasco-Dulbecco, Paul Cappeau. Identifier et caractériser un genre: l'exemple des interviews politiques. Langages, 2012, L'analyse de corpus face à l'hétérogénéité des données, 187, pp.27-40. 10.3917/lang.187.0027 . hal-00874048

\section{HAL Id: hal-00874048 \\ https://hal.science/hal-00874048}

Submitted on 19 Dec 2017

HAL is a multi-disciplinary open access archive for the deposit and dissemination of scientific research documents, whether they are published or not. The documents may come from teaching and research institutions in France or abroad, or from public or private research centers.
L'archive ouverte pluridisciplinaire HAL, est destinée au dépôt et à la diffusion de documents scientifiques de niveau recherche, publiés ou non, émanant des établissements d'enseignement et de recherche français ou étrangers, des laboratoires publics ou privés. 


\section{IDENTIFIER ET CARACTÉRISER UN GENRE : L'EXEMPLE DES INTERVIEWS POLITIQUES \\ Mylène Blasco-Dulbecco, Paul Cappeau}

Armand Colin | « Langages »

2012/3 n 187 | pages 27 à 40

ISSN 0458-726X

ISBN 9782200927769

Article disponible en ligne à l'adresse :

https://www.cairn.info/revue-langages-2012-3-page-27.htm

\section{Pour citer cet article :}

Mylène Blasco-Dulbecco, Paul Cappeau « Identifier et caractériser un genre : l'exemple des interviews politiques », Langages 2012/3 ( $\mathrm{n}^{\circ} 187$ ), p. 27-40. DOI 10.3917/lang.187.0027

Distribution électronique Cairn.info pour Armand Colin.

(C) Armand Colin. Tous droits réservés pour tous pays.

La reproduction ou représentation de cet article, notamment par photocopie, n'est autorisée que dans les limites des conditions générales d'utilisation du site ou, le cas échéant, des conditions générales de la licence souscrite par votre établissement. Toute autre reproduction ou représentation, en tout ou partie, sous quelque forme et de quelque manière que ce soit, est interdite sauf accord préalable et écrit de l'éditeur, en dehors des cas prévus par la législation en vigueur en France. Il est précisé que son stockage dans une base de données est également interdit. 


\section{Identifier et caractériser un genre : l'exemple des interviews politiques}

L'intérêt pour les genres a été relancé et en grande partie renouvelé par le développement de la linguistique de corpus (Biber 1988 ; Malrieu \& Rastier 2001 ; Cori \& David 2008). Cette notion, objet de constants réaménagements (Adam \& Heidmann 2006 ; Kerbrat-Orecchioni \& Traverso 2004 ; Bronckart 2008), ouvre des perspectives intéressantes pour la description des productions orales (BrancaRosoff 1999 ; Blanche-Benveniste 1997). Dans ce cadre, le genre s'écarte de la vision habituelle en littérature et correspond d'assez près à ce que $\mathrm{D}$. Biber et S. Conrad (2010) appellent registre ("a register is a variety associated with a particular situation of use", op. cit. : 6) dont la caractérisation peut être faite en termes de propriétés de langue ("registers are described for their typical lexical and grammatical characteristics: their linguistic features", ibid.). L'exploration de corpus d'interviews politiques à la radio soulève une série de questions : existe-t-il des faits de langue qui sont plus fortement sollicités dans un corpus d'interviews politiques et comment peut-on les identifier ? Quel tri opérer parmi les multiples faits morpho-syntaxiques présents dans un corpus : lesquels constituent de bons critères et comment s'assurer de leur pertinence ? Comment tenir compte de la dimension stylistique dans un corpus?

Cette réflexion sera présentée en trois étapes. Dans une première partie, nous préciserons les caractéristiques des corpus qui serviront d'appui à nos analyses. Dans la seconde, nous détaillerons un certain nombre de faits morphosyntaxiques et discuterons leur pertinence. Enfin, nous évoquerons l'influence du style propre à chaque locuteur. 


\section{PRÉSENTATION DU CADRE ET DES CORPUS}

Les corpus de langue parlée enregistrée dans les médias constituent, depuis quelques années, un champ d'étude à part entière. Ils ont donné lieu à de nombreuses analyses de type pragmatique (Andersen 2007, pour un exemple assez récent). Dans le domaine syntaxique, l'usage d'une langue médiatique a pu servir à décrire des changements en cours dans la norme (Blanche-Benveniste 2007 ; Branca-Rosoff 2007). L'orientation syntaxique adoptée dans cet article poursuit un autre objectif : proposer une réflexion méthodologique liée à la notion de genre. Plus précisément, il s'agit de déterminer si les interviews politiques constituent ou non un genre - le français parlé en contexte politico-médiatique auquel peut être reliée une panoplie de faits morpho-syntaxiques.

\subsection{Les corpus utilisés}

Dans une perspective de description linguistique sur corpus, le choix et la présentation de ces derniers sont importants. Nous nous appuyons sur des interviews d'hommes politiques dans les médias. Il s'agit d'une prise de parole que l'on peut qualifier de « mixte » : elle est à la fois publique (puisque portée devant un public réel de journalistes et d'invités et un auditoire virtuel, le public qui écoute l'émission) et professionnelle (cette situation de parole fait partie des compétences que doit maîtriser tout aspirant responsable de premier plan). Le dispositif utilisé habituellement dans ces émissions place l'interviewé « face » à des journalistes. La parole de l'homme politique est souvent hachée, entrecoupée par les interviewers, ce qui pose des difficultés pour isoler la seule parole de l'invité. Cette mise en scène vise à susciter l'intérêt de l'auditoire. La langue utilisée dans cette situation possède des caractéristiques orales plus manifestes que les discours et les interventions écrites produites par ces mêmes responsables dans d'autres situations (meetings, questions à l'Assemblée, etc.) qui ne sont oraux que par le médium utilisé (Koch \& Esterreicher 2001).

Nous disposons d'un corpus d'interviews d'hommes politiques (O-HP) dans les médias (enregistrés entre 1993 et 2003) qui compte 280000 mots ${ }^{1}$. S'en tenir à ces données présenterait des inconvénients majeurs : la taille du corpus est trop limitée pour espérer trouver de nombreux faits de langue en quantité suffisante ; d'autre part, repérer la présence d'une structure dans ce corpus ne peut garantir qu'il s'agit d'une propriété caractéristique. D'où la nécessité, afin de mieux cerner les éventuelles particularités de la langue parlée par les politiques dans les interviews, de recourir à d'autres corpus qui permettront d'effectuer des comparaisons éclairantes. Les corpus « adjacents » permettent de contraster, au mieux, les diverses composantes que l'on peut identifier dans les interviews politiques. Chaque corpus retenu présente la même taille (280 000 mots), ce qui donne au total un échantillon de langue de 1400000 mots, taille encore modeste,

1. Il s'agit d'émissions de radio comme Le Grand Jury sur RTL. Notre analyse porte sur le contenu verbal. 
mais qui permet d'observer la distribution de certains phénomènes. On aura ainsi recours aux corpus suivants ( $\mathrm{O}$ indique un corpus oral, $\mathrm{E}$ un corpus écrit) :

- O-PRI : il s'agit d'enregistrements en situation de parole privée appartenant au Corpus de Référence du Français Parlé ou CRFP (RSFP 18 ; Cappeau 2007). L'exploitation de ce corpus permettra de mieux identifier ce qui dans l'oral des politiques relève de la langue parlée habituelle ;

- O-PUB : il s'agit d'enregistrements en situation de parole publique (cours, conseil municipal, réunion, etc.) extraits du CRFP. Le but, en s'appuyant sur ce corpus, est de disposer d'échantillons d'une langue orale plus formelle ;

- E-DISC est un corpus écrit de discours politiques. Il semble opportun de travailler sur l'écrit car les interventions des politiques sont un lieu probable de croisement entre langue orale et langue écrite (cette dernière est mobilisée, par exemple, lors de la préparation des interviews, dans la mise au point de certaines formules et est aussi présente dans les diverses activités liées à cette activité professionnelle) ${ }^{2}$;

- E-PRESSE : enfin, il a semblé nécessaire de disposer de la langue journalistique (uniquement des textes liés au domaine politique) puisque la situation d'interviews met en présence hommes politiques et journalistes (la médiasphère).

\subsection{Le but du travail}

Attribuer la dénomination de corpus à un ensemble de transcriptions d'émissions de radio apparentées par la qualité des locuteurs relève d'un appui sur un critère extra-linguistique ${ }^{3}$. Cette décision demande à être validée par des spécificités de la langue. Nous sommes partis d'une hypothèse assez largement partagée depuis que les études sur corpus se sont développées (Biber 1988, entre autres) : celle de l'existence d'une relation assez étroite entre la situation de parole (externe) et des traits morpho-syntaxiques (internes). On fait l'hypothèse que le contenu lui-même des échanges intervient finalement assez peu sur les faits langagiers observés ${ }^{4}$. La question peut être précisée : le fait que l'échange soit médiatisé conduit-il le locuteur à recourir à des tournures syntaxiques particulières?

2. Une nouvelle piste, qui n'a pas été explorée, est ouverte par la situation contemporaine : la multiplicité des émissions auxquelles participent les interviewés. Il s'agirait alors d'observer les reprises importantes que les hommes politiques produisent d'une émission à l'autre. Une émission de Canal+ comme Le Petit journal pointe régulièrement ce procédé, qui brouille la frontière oral/écrit puisque ces passages réitérés correspondent souvent à des éléments de langage fournis par des communicants.

3. On pense ici à la distinction entre archive - simple collage de productions - et corpus - ensemble structuré (Rastier 2005 ; McEnery, Xia \& Tono 2006).

4. Voir la troisième partie pour quelques pistes portant sur la dimension individuelle, le style propre à chaque intervenant. 


\section{IDENTIFICATION DES INDICES PERTINENTS}

Deux séries d'indices morpho-syntaxiques peuvent servir à caractériser des productions : certains ont déjà fait l'objet de commentaires et servent généralement à typer une langue selon le trait plus ou moins formel, d'autres sont plus étroitement liés aux productions utilisées et émergent directement du corpus. Leur lien avec la situation d'interview s'avère plus sensible.

\subsection{Des faits généraux}

Les travaux sur grands corpus permettent de dégager des convergences de critères (en général morpho-syntaxiques) qui conduisent à proposer des classements et des regroupements de sous-corpus afin d'esquisser des typologies. À une échelle bien moindre (avec notamment un ensemble de formes réduit), cette analyse s'inscrira dans cette optique. Divers travaux, comme D. Biber (1988), ont mis en évidence la distribution singulière de certains éléments grammaticaux ${ }^{5}$. On les présente habituellement sous la forme de couples dans lesquels une unité apparaît comme banale (elle est fréquemment attestée dans l'usage courant), l'autre apparaît comme marquée (sa présence peut être corrélée à un trait comme le caractère formel ou soutenu de la production). Ainsi C. BlancheBenveniste (1997) précise-t-elle : « car au lieu de parce que ou nous à la place de on sont des variantes de prestige ». Trois couples ont été retenus à titre d'illustration. Ils serviront à présenter les enseignements et les limites des informations que peuvent apporter ces indices généraux.

\subsubsection{Car/Parce que}

On peut s'attendre, dans un discours qui relève (pour partie) de l'argumentation, à trouver des connecteurs tels que car ou parce que. Le relevé suivant permet de vérifier cette hypothèse :

Tableau 1 : Occurrences de car et parce que

\begin{tabular}{|l|c|c|c|c|c|}
\hline \multirow{2}{*}{} & \multicolumn{3}{|c|}{ ORAL } & \multicolumn{2}{c|}{ ECRIT } \\
\cline { 2 - 6 } & $\begin{array}{c}\text { HP } \\
\text { (interviews) }\end{array}$ & $\begin{array}{c}\text { PRI } \\
\text { (parole privée) }\end{array}$ & $\begin{array}{c}\text { PUB } \\
\text { (parole publique) }\end{array}$ & Presse & Discours \\
\hline car & 91 & 18 & 92 & 97 & 128 \\
\hline parce que & 780 & 1396 & 772 & 103 & 284 \\
\hline
\end{tabular}

La confrontation de nos cinq corpus apporte un éclairage supplémentaire et peut servir à identifier dans quelle configuration se place l'oral politique :

- le nombre d'occurrences de la conjonction car est assez comparable dans quatre des cinq corpus observés : seul, le corpus de parole privée (O-PRI) se distingue nettement de cet ensemble. Ce premier paramètre oriente vers une

5. La taille du corpus conduit à privilégier l'observation des faits grammaticaux même si, comme on le verra par la suite, certaines observations lexicales liées à des cooccurrences (Blanche-Benveniste 2010) peuvent être signalées. 
convergence, peu surprenante, entre notre corpus d'interviews et la parole publique, ainsi que les situations d'écrits retenues ;

- le nombre d'occurrences de la conjonction parce que apporte un éclairage légèrement différent qui conforte l'hypothèse que le corpus d'interviews est un objet complexe : la coupure est ici sensible entre corpus écrits et oraux d'une part et à l'intérieur des corpus oraux entre la situation de parole privée et les deux autres. On peut interpréter ce résultat comme l'indication que le corpus O-HP est manifestement un corpus oral ! Cette évidence demandait à être tout de même étayée puisque l'interview emprunte des traits à de nombreuses autres situations (ce que les corpus permettent d'établir). Mais il s'agit d'un oral néanmoins particulier : très proche de la parole publique et plutôt éloignée de la parole privée ;

- il resterait alors à mieux observer, dans le détail, la répartition des emplois de parce que entre l'introduction des commentaires (1), ce qui est fréquemment le cas dans l'oral privé (Debaisieux 2001) et la valeur véritablement causale (2) qui entre en relation avec la question pourquoi (3) ${ }^{6}$ :

(1) nous sommes revenus sur euh + sur la France moi euh avec beaucoup de + beaucoup de regrets parce que c'est vrai que j'aurais aimé rester plus longtemps (O-PRI)

(2) il a décliné le thème de de la sécurité et de l'insécurité bien entendu parce que c'est au cœur de la préoccupation des Français (O-HP)

(3) pourquoi a-t-il décliné le thème de la sécurité ? parce que c'est au cœur de la préoccupation des Français

\subsubsection{De sorte que/Pour que}

La présentation sous forme de couple est ici trompeuse. Les données font ressortir un tel déséquilibre que la comparaison n'est pas réellement satisfaisante : la locution de sorte que est si rare (quel que soit le corpus) qu'il devient illusoire de la retenir. Les comparaisons n'auraient plus grand sens :

Tableau 2 : Occurrences de de sorte que et pour que

\begin{tabular}{|l|c|c|c|c|c|}
\hline & \multicolumn{3}{|c|}{ ORAL } & \multicolumn{2}{c|}{ ECRIT } \\
\cline { 2 - 6 } & $\begin{array}{c}\text { HP } \\
\text { (interviews) }\end{array}$ & $\begin{array}{c}\text { PRI } \\
\text { (parole privée) }\end{array}$ & $\begin{array}{c}\text { PUB } \\
\text { (parole publique) }\end{array}$ & Presse & Discours \\
\hline de sorte que & 1 & 2 & 4 & 2 & 1 \\
\hline pour que & 129 & 96 & 136 & 54 & 58 \\
\hline
\end{tabular}

Seule, la conjonction pour que s'avère un indice intéressant : elle fait ressortir que le corpus oral politique est proche de celui de parole publique et qu'il s'écarte des autres productions. Il faudrait pousser plus avant le fonctionnement syntaxique de cette conjonction pour mieux apprécier le rapprochement que les

6. Les exemples oraux sont transcrits en orthographe, sans majuscule ni ponctuation. Les '+' correspondent à des pauses et les mots amorcés sont signalés par un trait d'union en position finale. 
résultats quantitatifs esquissent et observer si les fonctionnements syntaxiques sont comparables :

(4) un signe de changement assez fort pour que nul ne puisse plus l'ignorer (O-HP)

(5) il a fallu dix minutes pour que + je réussisse à à ne plus tétaniser (O-PRI)

(6) Depuis, elles ont décidé de se battre pour que d'autres enfants ne meurent pas de ce jeu. (E-Presse)

\subsubsection{Par contre/En revanche}

Ce dernier couple se retrouve dans de nombreuses grammaires. La locution par contre est depuis fort longtemps stigmatisée par les puristes et l'oral politique reflète bien cette réalité :

Tableau 3 : Occurrences de par contre et en revanche

\begin{tabular}{|l|c|c|c|c|c|}
\hline \multirow{2}{*}{} & \multicolumn{3}{|c|}{ ORAL } & \multicolumn{2}{c|}{ ECRIT } \\
\cline { 2 - 6 } & $\begin{array}{c}\text { HP } \\
\text { (interviews) }\end{array}$ & $\begin{array}{c}\text { PRI } \\
\text { (parole privée) }\end{array}$ & $\begin{array}{c}\text { PUB } \\
\text { (parole publique) }\end{array}$ & Presse & Discours \\
\hline par contre & 7 & 61 & 64 & 3 & 5 \\
\hline en revanche & 23 & 1 & 4 & 33 & 10 \\
\hline
\end{tabular}

L'oral politique se révèle sensible à l'influence de la norme écrite et s'écarte des usages oraux (tant de la parole privée que publique) : par contre y est rare (7), en revanche y est d'un usage relativement important $(8)^{7}$ :

(7) ce que j'attends par contre du gouvernement c'est qu'il hiérarchise ses priorités (O-HP)

(8) c'est un homme à la vie privée qui n'a pas été heureuse mais en revanche il a eu une grande histoire d'amour (O-HP)

Avec cette première série de faits souvent signalés, la confrontation des cinq corpus valide des tendances connues concernant la répartition des formes. Elle apporte toutefois des indications supplémentaires sur la représentation de la langue formelle, des nuances sur la spécificité de l'usage oral dans les interviews politiques ainsi qu'une réflexion sur la pertinence des critères utilisés. Mais sur ces points connus les avancées restent peu spectaculaires, d'où la nécessité de chercher des phénomènes plus saillants qui font ressortir l'originalité de la langue utilisée dans les interviews.

\subsection{Des faits plus spécifiques}

Le travail sur corpus permet de faire émerger des faits moins prévisibles, étroitement liés aux données. Cela suppose de s'appuyer certes sur des données

7. Les faits observés dans les corpus condamnent sans appel l'hypothèse naïve et obsolète qui présente en revanche comme une forme de compensation de par contre. 
quantitatives, mais aussi de décrire plus précisément les emplois que ces éléments grammaticaux peuvent avoir. Notre réflexion portera sur certains faits de langue que la situation d'interview tend à favoriser. Il s'agit là d'illustrer comment peuvent être recherchés et proposés des indices pertinents. On s'attend ainsi à ce que les interventions d'hommes politiques soient assez fortement structurées ( $d$ 'où le choix d'observer plus attentivement les connecteurs utilisés dans la planification de la production). On suppose aussi que le locuteur doit développer des stratégies pour conserver la parole et prendre le temps de réfléchir aux réponses à apporter (dans le domaine verbal, cela semble passer par le biais de tournures syntaxiques longues).

\section{a) Les marqueurs de segmentation}

Tout oral de débat laisse attendre la présence de marqueurs de segmentation qui vont servir à organiser et planifier la production (Adam \& Revaz 1989). On s'en tiendra à l'examen de d'une part et d'autre part, attestés dans l'ensemble des corpus $^{8}$ :

Tableau 4 : Occurrences de d'une part et d'autre part

\begin{tabular}{|l|c|c|c|c|c|}
\hline \multirow{2}{*}{} & \multicolumn{3}{|c|}{ ORAL } & \multicolumn{2}{c|}{ ECRIT } \\
\cline { 2 - 6 } & $\begin{array}{c}\text { HP } \\
\text { (interviews) }\end{array}$ & $\begin{array}{c}\text { PRI } \\
\text { (parole privée) }\end{array}$ & $\begin{array}{c}\text { PUB } \\
\text { (parole publique) }\end{array}$ & Presse & Discours \\
\hline d'une part & 16 & 13 & 12 & 4 & 14 \\
\hline d'autre part & 27 & 4 & 32 & 10 & 18 \\
\hline
\end{tabular}

Plusieurs observations peuvent être tirées de ces données. Le fonctionnement corrélé de ces marqueurs est mis à mal par ces résultats, ce qui est bien connu : d'une part est, de fait, moins utilisé que d'autre part dans la plupart des corpus (seule la parole privée diffère nettement sur ce point). Ce déséquilibre (observable aussi à l'écrit) tient au fait que chacun de ces connecteurs possède des valeurs et des emplois très variés et entre dans des oppositions multiples. Ainsi, en (9) d'une part s'oppose à de notre côté, dans (10) d'autre part sert à introduire un nouvel argument :

(9) il faut que les politiques d'une part reconnaissent qu'ils sont pas euh les seuls euh à décider qu'il y a donc cette société civile et c'est pour ça qu'il faut que le gouvernement actuel rentre dans une euh refonte euh du dialogue social dans une rénovation é- et pour changer les règles ça c'est le rôle des politiques les syndicats de notre côté il faut qu- qu'on s'applique à nous euh les reproches $(\mathrm{O}-\mathrm{HP})$

(10) L1 est-ce que vous ne privilégiez pas le court terme au détriment du long terme

L2 non parce que euh vous savez si vous avez escompté une recette mais qu'elle ne se présente pas euh c'est une fausse recette d'autre part comme

8. La taille réduite du corpus a quelque peu orienté la sélection des formes retenues. Les formes les plus nombreuses ont été privilégiées pour ce travail. 
vous l'avez souligné il y aura des recettes de substitution qu'il s'agisse d'autoroutes du sud de la France [...] (O-HP)

Les deux connecteurs ne présentent pas le même intérêt dans notre réflexion sur le genre. Le premier (d'une part) s'avère peu révélateur puisqu'il se rencontre avec un nombre d'occurrences proches, plutôt faible, dans la plupart des productions utilisées. Le second (d'autre part) se démarque davantage par sa répartition. Il peut donc être considéré comme un indice pertinent : il conduit à singulariser le corpus d'interviews et permet d'établir, à nouveau, une proximité entre les interviews et la parole publique.

Il faudrait certainement mieux analyser les emplois de ces connecteurs et élargir le paradigme à d'autres marqueurs tels que par ailleurs, de plus, en outre, entre autres, pour disposer de plus de traits distinctifs du corpus d'interviews.

\section{b) Les formes de remplissage}

Les interviews politiques comportent quelques formes grammaticales originales présentant un format long inhabituel, qui ont été identifiées à la suite d'une observation plus générale des unités grammaticales dans ce corpus d'interviews. On prendra deux exemples : l'un lié au déterminant, l'autre aux relatives.

Dans le cas de l'expression du déterminant indéfini, on rencontre la suite un certain nombre de qui présente la répartition suivante dans les corpus :

Tableau 5 : Occurrences de un certain nombre de

\begin{tabular}{|l|c|c|c|c|c|}
\hline & \multicolumn{3}{|c|}{ ORAL } & \multicolumn{2}{c|}{ ECRIT } \\
\cline { 2 - 6 } & $\begin{array}{c}\text { HP } \\
\text { (interviews) }\end{array}$ & $\begin{array}{c}\text { PRI } \\
\text { (parole privée) }\end{array}$ & $\begin{array}{c}\text { PUB } \\
\text { (parole publique) }\end{array}$ & Presse & Discours \\
\hline $\begin{array}{l}\text { un certain } \\
\text { nombre de }\end{array}$ & 139 & 20 & 68 & 10 & 70 \\
\hline
\end{tabular}

Cette forme fait ressortir le caractère atypique des interviews : les occurrences de ce déterminant long y sont deux fois plus nombreuses que dans le corpus de discours écrits. En ce sens, il constitue un marqueur identificatoire précieux ${ }^{9} \mathrm{du}$ genre interviews politiques :

(11) et donc il fallait prendre un certain nombre de dispositions (O-HP)

(12) le président incarne un certain nombre de valeurs qui sont celles de notre nation (O-HP)

Ce déterminant possède d'autres avantages qui apparaissent par comparaison avec des formes concurrentes comme plusieurs (13) et beaucoup de (14) :

(13) j'ai eu des contacts avec plusieurs personnalités américaines (O-HP)

(14) et je trouve qu'il a fait beaucoup de choses en quatre mois (O-HP)

9. Pour être plus précis, un seul indicateur n'est pas pleinement satisfaisant. De fait, c'est bien la convergence de marqueurs morpho-syntaxiques qui fait sens. 
Ces deux derniers déterminants se révèlent moins adaptés à la situation d'interview comme en attestent les alternatives proposées à l'exemple (15). Le recours à plusieurs (16) ouvre la possibilité d'une demande de précision de type combien ? ou lesquelles? L'utilisation d'une forme comme beaucoup de (17) serait trop orientée vers la quantité et s'avérerait moins efficace (car potentiellement plus risquée pour le locuteur qui pourrait être conduit à préciser sa position). Finalement, un certain nombre de apparaît comme une formule précautionneuse bien adaptée à la situation :

on ne peut pas évidemment augmenter l'action de la police sans en tirer un certain nombre de conséquences (O-HP)

on ne peut pas évidemment augmenter l'action de la police sans en tirer plusieurs conséquences

on ne peut pas évidemment augmenter l'action de la police sans en tirer beaucoup de conséquences

Notre hypothèse sur la fonction discursive du déterminant long est confortée par la comparaison du nombre d'occurrences de ces trois formes : un certain nombre de est effectivement plus employé que plusieurs et beaucoup de dans les interviews politiques. C'est d'ailleurs le seul corpus où cette tendance peut être observée. Par ailleurs, une autre propriété convergente est ouverte par le choix des items lexicaux qui suivent les divers déterminants. La suite un certain nombre de (18) s'adapte à un lexique varié (indice de sa malléabilité argumentative), les deux autres déterminants présentent de nombreux emplois plus ciblés : plusieurs (19) est lié à des noms temporels (années, fois, ...), beaucoup de (20) à des termes plus génériques (gens, choses, ...) :

(18) le chef de l'État est obligé de euh répondre un certain nombre de contraintes budgétaires (O-HP)

(19) j'ai réagi lorsqu'il a été question de différer de plusieurs années la baisse des impôts (O-HP)

(20) il y a beaucoup de gens qui ont senti se sont sentis menacés (O-HP)

Les indications quantitatives et les propriétés distributionnelles convergent et permettent $d$ 'identifier un critère morpho-syntaxique pertinent parmi plusieurs faits concurrents.

M. Halliday (1985) a bien montré que l'une des lignes de partage entre l'écrit et l'oral tenait à la place qu'occupaient certaines catégories. La proposition relative illustre une relation entre les contraintes dues à la situation d'interview et les formes syntaxiques employées. C. Blanche-Benveniste (1995) ou P. Cappeau (2003) ont observé certains emplois spécifiques des relatives sensibles aux types de productions. Il existe, dès lors que l'on y regarde de près, de nombreuses sortes de relatives (Gapany 2004) qui permettent de proposer des caractérisations plus fines. Ainsi, dans les productions politiques, on rencontre trois types de relatives singulières. Elles peuvent être schématisées par les séquences qui est celui (21), un N1 qui est un N1 (22) et qui est le mien (23) : nous nous inscrivons dans une tradition qui est celle de la France (O-HP) 
$c^{\prime}$ est un accord qui est un accord global (O-HP)

(23) je suis très heureux de pouvoir montrer du pays qui est le mien et que j'aime un visage attrayant (O-HP)

Ces relatives sont inégalement présentes dans les corpus :

Tableau 6 : Occurrences de diverses relatives

\begin{tabular}{|l|c|c|c|c|c|}
\hline & \multicolumn{3}{|c|}{ ORAL } & \multicolumn{2}{c|}{ ECRIT } \\
\cline { 2 - 6 } & $\begin{array}{c}\text { HP } \\
\text { (interviews) }\end{array}$ & $\begin{array}{c}\text { PRI } \\
\text { (parole privée) }\end{array}$ & $\begin{array}{c}\text { PUB } \\
\text { (parole publique) }\end{array}$ & Presse & Discours \\
\hline qui est celui & 10 & 0 & 8 & 1 & 11 \\
\hline qui est le mien & 19 & 0 & 0 & 0 & 4 \\
\hline N1 qui est un N1 & 8 & 0 & 4 & 0 & 4 \\
\hline
\end{tabular}

Sans conteste, l'interview politique se singularise par l'exploitation de ces structures : elles ne sont présentes ni dans l'oral tout venant (O-PRI) ni dans la presse (E-PRESSE). La relative qui est celui établit une proximité, déjà observée, entre interview politique et parole publique ainsi qu'avec les discours écrits. Il est surprenant de voir que, malgré le poids de la tradition normative en France, le discours politique s'éloigne, sur ce point, radicalement des schémas de l'écrit standard. Chacune de ces structures apporte donc son propre éclairage sur la délimitation du genre « interview politique » qui apparaît au fil des résultats obtenus dans ce travail comme une notion non seulement complexe, mais surtout hétérogène (qui selon les paramètres utilisés s'éloigne ou s'écarte plus ou moins des corpus adjacents qui ont été retenus).

La tentation est grande de considérer que ces relatives dont on pourrait penser, au premier abord, qu'elles ne disent rien (Noailly \& Richard 2007) ont pour seul but de meubler, de maintenir la prise de parole. Leur valeur se révèle mieux lorsqu'on les contraste avec des formes concurrentes. Ainsi, la confrontation entre la relative du type qui est le mien et le déterminant possessif engage à modifier la première impression. Une tournure telle que ce pays qui est le mien conduit à différer l'expression de la relation d'appartenance et à présenter en premier une forme non marquée par le lien de possession (relatif qui + copule est). Il semble que, par ce biais, le locuteur adopte une prise de position atténuée qui est plus habile dans la situation d'échange. Cette relative est d'ailleurs assez souvent décrochée sur le plan prosodique et s'apparente plutôt à une parenthèse. Elle introduit une hiérarchie dans l'information délivrée par l'ensemble de la séquence. Dans l'exemple (24), le locuteur a la possibilité de placer (par la prosodie) au second plan qui a été le mien et ainsi d'atténuer son implication. À l'inverse, l'emploi du déterminant possessif comme dans la tournure mon pays (25) peut paraître trop direct (voire passionnel) et s'avérer potentiellement plus clivant :

(24) jugez le comportement qui a été le mien à l'aune de votre réflexion $(\mathrm{O}-\mathrm{HP})$

(25) je considérais que c'était une insulte pour mon pays (O-HP) 
L'observation du lexique qui suit le déterminant possessif le confirme : on trouve des termes tels que avis, sentiment, question, position qui tous reflètent une prise de position assumée, défendue par le locuteur (26) et (27) :

et sur ce point-là ma position est claire je suis pour que l'État demeure majoritaire (O-HP)

ce n'est pas à moi de dire si untel ou untel a bien ou mal géré telle ou telle entreprise publique ça n'est pas mon affaire (O-HP)

Avec la relative, la présentation est bien plus distanciée, du moins en apparence. En (28), le locuteur avance masqué pour présenter un point de vue qui semble introduit de façon neutre :

il y a une autre vision qui est la mienne qui dit vous ne pouvez pas priver les citoyens du droit de dire leur mot (O-HP)

Avec les relatives, le discours politique utilise une syntaxe qui remplace le déterminant et l'adjectif par une structure verbale qui alourdit le syntagme nominal (mon pays / le pays qui est le mien; un accord global / un accord qui est global ; une tradition française / une tradition qui est celle de la France communauté de citoyens). Ce pourrait être une construction progressive du nom, en deux étapes, une sorte de stratégie discursive pour argumenter, prendre soin d'avancer sans bousculer.

Même si le nombre de critères qui ont été traités reste réduit, les résultats obtenus peuvent être considérés comme probants ou, du moins, comme une orientation prometteuse : ils permettent réellement de dégager des usages de certaines tournures syntaxiques fortement dépendants de la situation d'échange propre aux interviews politiques. En l'état, les indices que nous avons examinés orientent vers l'hypothèse que les interviews politiques constituent bien un genre oral. Toute une série de faits convergent vers cette piste : les données quantitatives, les faits de distribution plus précis, la comparaison du fonctionnement de formes concurrentes, les associations lexicales. Ce matériau linguistique contient des outils grammaticaux adaptés à un genre (l'interview politique) où conserver la parole et développer une argumentation constituent des enjeux importants et influent sur la construction du discours.

Il reste à envisager un angle complémentaire qui peut limiter ces orientations : l'importance à accorder aux dimensions individuelles.

\section{LES VARIATIONS INDIVIDUELLES}

Cette partie a pour but d'attirer l'attention sur des problèmes difficiles à intégrer dans des approches quantitatives sur corpus : le lien entre les productions et les locuteurs. Il est souvent difficile, si l'on s'en tient à la seule observation des fréquences, de faire la part entre une propriété partagée par tous et un idiolecte. Ainsi, l'expression ce pays qui est le mien se rencontre chez François Bayrou dans un premier corpus, puis sous la forme ce pays qui est le sien dans une autre 
émission avec le même locuteur. On voit, sur ce simple exemple et à petite échelle, à quel point il faut être attentif notamment lorsque l'on travaille sur des faits grammaticaux ou lexicaux faiblement représentés et sur des corpus de taille réduite. Un autre aspect doit être précisé et tient directement à la situation externe qui a été utilisée : les émissions politiques sont le résultat d'échanges entre hommes politiques et journalistes. Des passerelles entre le discours écrit de presse et le corpus d'interviews peuvent être dégagées. Cette parenté ne tientelle pas aux locuteurs impliqués dans la situation ? En l'occurrence, les faits examinés se rattachent plutôt aux locuteurs politiques (comme l'a montré leur fonction), mais il pourrait être intéressant de prolonger la réflexion et de mieux mesurer l'influence que la langue des journalistes peut avoir sur la parole des politiques.

Un lien étroit entre le contenu et les formes grammaticales utilisées peut être observé chez Jacques Delors lorsqu'il annonce qu'il ne sera pas candidat à l'élection présidentielle de 1995. Il enchaîne une série d'interrogatives - série que l'on ne retrouvera pas ailleurs - qui reflètent probablement ses hésitations et son renoncement final :

(29) L1 c'est pour ça d'ailleurs qu'il y a eu des disputes inutiles quels sont les problèmes des générations à venir de ceux qui vont gouverner maintenant c'est comment concilier l'extension de nos valeurs de paix de reconnaissance mutuelle entre nous qui est quand même qui sont quand même des acquis de la construction européenne comment concilier ces valeurs avec l'élargissement à vingt-sept voire trente pays comment le faire $(\mathrm{O}-\mathrm{HP})$

Les productions orales de François Bayrou se caractérisent par un nombre important de reformulations et par l'expansion de certains constituants (30)(32). En apparence, ces séquences devraient nuire à la clarté et à l'efficacité de l'intervention. En fait, ne contribuent-elles pas à forger un style et par là une caractérisation du locuteur?

(30) je pense qu'il y a deux choses qui sont manifestes ou manifestement symétriques ou parallèles entre le temps d'Henri IV et le nôtre (O-HP)

$c^{\prime}$ est que le seizième siècle il est entièrement déstabilisé par l'intervention d'un média nouveau qui est l'imprimerie qui donne aux gens l'accès direct au texte qui donc ruine l'autorité qui jusqu'alors transmettait seule les textes (O-HP)

c'était en même temps un homme privé dont je crois qu'il a été beaucoup moins heureux qu'on ne le dit euh mais ne se laissant jamais détourner par ses propres difficultés intérieures euh ne s'en laissant jamais détourner de rire $(\mathrm{O}-\mathrm{HP})$

Les variations propres aux locuteurs orientent vers la vaste problématique des styles (Rastier 2001 ; Gadet 2004 ; Biber \& Conrad 2010), sujet particulièrement intéressant dans le cadre des productions orales (Bilger \& Cappeau 2004). L'observation des faits de langue par l'entrée individuelle ouvre d'autres perspectives encore trop peu explorées. 


\section{CONCLUSION}

Les résultats présentés s'inscrivent dans le cadre d'une étude comparative sur corpus. Du point de vue méthodologique, ils montrent que l'on peut conduire des analyses sur des corpus de taille modeste dès lors que l'on parvient à intégrer une certaine variété dans les données : il était bon de confronter les oraux des politiques en interview à d'autres formes de production tant orales qu'écrites pour délimiter les spécificités de ce type de parole. Le français parlé en contexte politico-médiatique se constitue bien en empruntant des traits à la parole privée (rarement), à la parole publique (assez régulièrement) et à l'écrit. En ce sens, il constitue un genre spécifique et hétérogène. Les données nous ont permis de trier parmi les formes morpho-syntaxiques celles qui sont peu exploitables (par leur quantité ou par leur répartition homogène dans tous les corpus) et celles que l'on peut considérer comme probantes (parce qu'elles permettent de singulariser le corpus d'interviews). Cette démarche conduit à réviser certains a priori sur la langue (la langue orale des politiques se rapproche parfois de la langue publique et pas nécessairement de la langue écrite, elle se différencie d'ailleurs assez souvent de la langue écrite de presse) et à découvrir des tournures originales (la forme du déterminant un certain nombre de ou de certaines relatives). Une observation plus fine du fonctionnement de ces unités a montré un lien, en partie inattendu, avec la situation externe, une sorte de spécialisation de certains outils de la langue qui ne recoupe pas la notion de langue de bois, généralement accolée aux politiques.

\section{Références}

[RSFP] Recherches Sur le Français Parlé n 18 : Autour du Corpus de Référence du Français Parlé, Aix-en-Provence : Presses Universitaires de Provence, 2004.

Adam J.-M. \& Heidmann U. (2006), "Propositions pour l'étude de la généricité ", La Licorne 79, 21-34.

AdAM J.-M. \& REVAZ F. (1989), "Aspects de la structuration du texte descriptif : les marqueurs d'énumération et de reformulation ", Langue française 81, 59-98.

ANDERSEN H. L. (2007), "L'interview comme genre médiatique : sous-catégories pragmatiques et leurs traits linguistiques caractéristiques ", in M. Broth et al. (éds), Le français parlé des médias, Stockholm : Acta Universitatis Stockholmiensis, 153-172.

BIBER D. (1988), Variation across speech and writing, Cambridge: Cambridge University Press.

Biber D. \& ConRAD S. (2010), Register, Genre, and Style, Cambridge: Cambridge University Press.

BILger M. \& CAPPEAU P. (2004), " L'oral ou la multiplication des styles ", Langage et société 109, 13-30.

BLANCHE-BENVENISTE C. (1995), " De la rareté de certains phénomènes syntaxiques en français parlé ", Journal of French Language Studies 5, 17-29.

BlanCHE-BenVENISTE C. (1997), "La notion de variation syntaxique dans la langue parlée ", Langue française 115, 19-29. 
Blanche-BenVeniste C. (2007), " Normes anciennes et nouvelles dans le langage des médias ", in M. Broth et al. (éds), Le français parlé des médias, Stockholm : Acta Universitatis Stockholmiensis, 31-48.

Blanche-BenVEniste C. (2010), Le français : Usages de la langue parlée, Leuven : Peeters.

BRANCA-Rosoff S. (1999), "Des innovations et des fonctionnements de langue rapportés à des genres ", Langage et Société 87, 115-129.

BrancA-Rosoff S. (2007), "Nouveaux genres et déplacement de normes en français. À propos des interviews politiques sur les radios généralistes et des émissions de 'libre antenne' sur les radios-jeunes ", in M. Broth et al. (éds), Le français parlé des médias, Stockholm : Acta Universitatis Stockholmiensis, 63-82.

BRonckart J.-P. (2008), "Genres de textes, types de discours et "degrés” de langue : hommage à François Rastier ", Texto! [http://www.revue-texto.net/docannexe/file/86/bronckart_rastier.pdf]

CAPPEAU P. (2003), "Vous mélangez tout ? L'ordre dans les recettes ", in B. Combettes, C. Schnedecker \& A. Theissen (éds), Ordre et distinction dans la langue et le discours, Paris : Champion, 87-99.

CAPPEAu P. (2007), "Constituer et exploiter un gros corpus oral : l'exemple du CRFP ", in M. Ballard \& C. Pineira-Tresmontant (éds), Les corpus en linguistique et en traductologie, Arras : Artois Presses Université, 145-158.

CoRI M. \& DAVID S. (2008), " Les corpus fondent-ils une nouvelle linguistique ? ", Langages 171, 111-129.

DEBAISIEUX J.-M. (2001), " Le fonctionnement de parce que en français parlé : étude quantitative sur corpus ", in C. D. Pusch \& W. Raible (eds), Romanistische Korpuslinguistik - Korpora und gesprochene Sprache, Romance Corpus Linguistics, Corpora and Spoken Language, Tübingen : Gunter Narr Verlag, 349-362.

GADET F. (2004), "Le style comme perspective sur la dynamique des langues ", Langage et société 109, 1-8.

GAPANY J. (2004), Formes et fonctions des relatives en français. Étude syntaxique et sémantique, Bern : Peter Lang.

HaLLIDAY M. A. K. (1985), Spoken and Written Language, Oxford: University Press.

Kerbrat-Orecchioni C. \& Traverso V. (2004), "Types d'interactions et genres de l'oral ", Langages 153, 41-51.

KOCH P. \& OESTERREICHER W. (2001), " Langage oral et langage écrit ", Lexikon der romanistischen Linguistik, Tome 1/2, Tübingen : Max Niemeyer Verlag, 584-627.

MALRIEU D. \& RASTIER F. (2001), "Genres et variations morphosyntaxiques ", Traitement Automatique des Langues 42 (2), 548-577.

McEnery T., XIA R. \& Tono Y. (2006), Corpus-Based Language Studies. An advanced resource book, New York: Routledge Applied Linguistics.

NoAilly M. \& RichaRd E. (2007), "Des relatives pour ne (presque) rien dire ", in M. Broth et al. (éds), Le français parlé des médias, Stockholm : Acta Universitatis Stockholmiensis, 567-578.

RASTIER F. (2001), "Vers une linguistique des styles ", L'Information grammaticale 89, 3-6.

RASTIER F. (2005), "Enjeux épistémologiques de la linguistique de corpus ", in G. Williams (éd.), La linguistique de corpus, Rennes: Presses Universitaires de Rennes, 31-45. 